\title{
A NOTE ON COMPLETELY METRIZABLE SPACES
}

\author{
E. MICHAEL
}

\begin{abstract}
Completely metrizable spaces are characterized by means of certain not necessarily open covers, and some applications are given to the preservation of complete metrizability under mappings.
\end{abstract}

1. Introduction. The purpose of this note is to obtain some new characterizations of completely metrizable spaces ${ }^{1}$ by means of certain covers which are not necessarily open, and to give some applications. Our investigation originally grew out of an analysis of a recent result of $\mathrm{N}$. Ghoussoub and B. Maurey in [GM], and I am grateful to my colleague Isaac Namioka for calling a preprint of that paper to my attention.

Completely metrizable spaces have been characterized both externally and internally. The most useful external characterization, due to P. Alexandrov $[\mathbf{A}]$ and F. Hausdorff $\left[\mathbf{H}_{\mathbf{1}}\right]$, is simple and well-known.

THEOREM $1.1\left[\mathbf{A}, \mathbf{H}_{1}\right]$. A metrizable space $X$ is completely metrizable if and only if it is a $G_{\delta}$-subset of a complete metric space.

To state the most familiar internal characterization, we make the following definition. Call a sequence $\left(\mathscr{U}_{n}\right)$ of covers of a space $X$ complete if, whenever $\mathscr{F}$ is a filter base on $X$ such that each $\mathscr{U}_{n}$ has an element $U_{n}$ containing some $F \in \mathscr{F}$, then $\bigcap\{\bar{F}: F \in \mathscr{F}\} \neq \varnothing .^{2}$ The following result was obtained by Z. Frolik [F] and A. V. Arhangel'skiı̌ [Ar].

TheOREM 1.2 [F, Ar]. A metrizable space $X$ is completely metrizable if and only if it has a complete sequence of open covers.

Various modifications of Theorem 1.2 are known (see, for example, [We] and the result quoted in Theorem 1.4 below), but all of these are in terms of open covers. The first purpose of this note is to give (in Theorem 1.3) a new and weaker characterization in terms of covers which need not be open.

Let us call a cover $\mathscr{U}$ of a space $X$ exhaustive if every nonempty $S \subset X$ has a nonempty, relatively open subset of the form $U \cap S$ with $U \in \mathscr{U}$. Clearly every open

Received by the editors October 22, 1984 and, in revised form, March 15, 1985.

1980 Mathematics Subject Classification. Primary 54E50, 54C10, 54E99.

Key words and phrases. Completely metrizable, complete, exhaustive, sieve, topological game.

${ }^{1}$ I.e., Metrizable with a complete metric.

${ }^{2}$ Analogously, one can define a countably complete sequence of covers by restricting $\mathscr{F}$ to be a countable filter base, and a similar modification can be made for sieves. Theorems $1.2-1.5$ remain true with "complete" weakened to "countably complete". 
cover of $X$ is exhaustive, and so is the cover $\{\{x\}: x \in X\}$ of $X$ if $X$ is a scattered ${ }^{3}$ space.

THEOREM 1.3. A metrizable space is completely metrizable if and only if it has a complete sequence of exhaustive covers.

Theorem 1.3 immediately implies the well-known result that every scattered metrizable space is completely metrizable; it suffices to let $\mathscr{U}_{n}=\{\{x\}: x \in X\}$ for all $n$ in Theorem 1.3.

The characterizations in Theorems 1.2 and 1.3, while simple and elegant, are not sufficiently flexible for some applications, such as the preservation of complete metrizability under certain kinds of maps. We therefore introduce the following concept (see [C ČN, M]).

A sieve on a space $X$ is a sequence of indexed covers $\left\{U_{\alpha}: \alpha \in A_{n}\right\}(n \geqslant 0)$ of $X,{ }^{4}$ together with maps $\pi_{n}: A_{n+1} \rightarrow A_{n}$, such that $U_{\alpha}=X$ for $\alpha \in A_{0}$ and $U_{\alpha}=$ $\bigcup\left\{U_{\beta}: \beta \in \pi_{n}^{-1}(\alpha)\right\}$ for all $\alpha \in A_{n}$ and all $n$. Such a sieve is called complete if, whenever $\alpha_{n} \in A_{n}$ with $\pi_{n}\left(\alpha_{n+1}\right)=\alpha_{n}$ for all $n$, and whenever $\mathscr{F}$ is a filter base on $X$ such that each $U_{\alpha_{n}}$ contains some $F \in \mathscr{F}$, then $\bigcap\{\bar{F}: F \in \mathscr{F}\} \neq \varnothing$.

The following modification (and strengthening) of Theorem 1.2 was obtained in [C ČN and M]. We call a sieve $\left(\left\{U_{\alpha}: \alpha \in A_{n}\right\}, \pi_{n}\right)$ on $X$ an open sieve if every $U_{\alpha}$ is open in $X$.

THEOREM 1.4 [ČČN, M]. A metrizable space $X$ is completely metrizable if and only if it has a complete open sieve.

We now come to an analogous modification of Theorem 1.3. Call a sieve ( $\left.\left\{U_{\alpha}: \alpha \in A_{n}\right\}, \pi_{n}\right)$ on $X$ an exhaustive sieve if $\left\{U_{\beta}: \beta \in \pi_{n}^{-1}(\alpha)\right\}$ is an exhaustive cover of $U_{\alpha}$ for all $\alpha \in A_{n}$, and all $n .^{5}$ Observe that every open sieve is clearly exhaustive.

THEOREM 1.5. A metrizable space $X$ is completely metrizable if and only if it has a complete exhaustive sieve.

As observed, for example, in [M], Theorem 1.4 immediately implies the theorem of F. Hausdorff $\left[\mathbf{H}_{2}\right]$ that, if $f: X \rightarrow Y$ is a continuous, open map from a completely metrizable space $X$ onto a metrizable space $Y$, then $Y$ is also completely metrizable. Indeed, if $\left(\left\{U_{\alpha}: \alpha \in A_{n}\right\}, \pi_{n}\right)$ is a complete open sieve on $X$, then $\left(\left\{f\left(U_{\alpha}\right): \alpha \in A_{n}\right\}, \pi_{n}\right)$ is a complete open sieve on $Y$. The simplicity of this argument contrasts strikingly with Hausdorff's complicated and lengthy proof in $\left[\mathbf{H}_{2}\right]$. In explanation, it should be remarked that the proof of Theorem 1.4 (see [M,

\footnotetext{
${ }^{3}$ Recall that a space is scattered if every nonempty subset has an isolated point.

${ }^{4}$ The index sets $A_{n}$ are assumed to be disjoint. Unlike [C $\check{C} \mathbf{N}$ and $\mathbf{M}$ ], we do not assume that the sets $U_{a}$ are open in $X$.

${ }^{5}$ This implies that $\left\{U_{\alpha}: \alpha \in A_{n}\right\}$ is an exhaustive cover of $X$ for all $n$. As a partial converse, if $\left(\left\{U_{\alpha}\right.\right.$ : $\left.\left.\alpha \in A_{n}\right\}, \pi_{n}\right)$ is a sieve on $X$ such that $\left\{U_{\alpha}: \alpha \in A_{n}\right\}$ is a disjoint exhaustive cover of $X$ for all $n$, then it is an exhaustive sieve on $X$.
} 
p. 721]) uses the paracompactness of metrizable spaces, and that fact was, of course, not available to Hausdorff in 1934.

The result of the previous section was extended in $[\mathbf{M}]$ to a larger class of maps, called tri-quotient maps, which includes both continuous open maps and perfect maps. Using Theorem 1.5, we can now enlarge the class of maps even further. We call a metric space $\varepsilon$-modest if it is the union of finitely many subsets of diameter $\leqslant \varepsilon$.

TheOREM 1.6. Let $f: X \rightarrow Y$ be a continuous map from a complete metric space $X$ onto a metrizable space $Y$. Suppose $X$ has a cover $\mathscr{U}$ such that $X \in \mathscr{U}$ and, if $U \in \mathscr{U}$ and $\varepsilon>0$, then

$$
\{f(V): V \in \mathscr{U}, V \subset U, V \text { is } \varepsilon \text {-modest }\}
$$

is an exhaustive cover of $f(U)$. Then $Y$ is completely metrizable.

The requirement in Theorem 1.6 is clearly satisfied by open maps (take $\mathscr{U}$ to be the collection of open subsets of $X$ ) and, more generally, by tri-quotient maps [M, §6] (take $\mathscr{U}=\left\{U \subset X: U\right.$ open, $\left.U^{*}=f(U)\right\}$ ). It is also satisfied by the following class of (not necessarily quotient) maps.

Call a map $f: X \rightarrow Y$, with $X$ a metric space, spacious if, for every $\varepsilon>0$ and every $\varepsilon$-discrete ${ }^{6} A \subset X$, the space $f(A)$ has an isolated point (which implies that $f(A)$ must actually be scattered). For example, a map $f: X \rightarrow Y$ with metric $X$ is surely spacious if $f$ is a closed map or if $Y$ is a scattered space. As we shall see in Lemma 6.1, a map $f: X \rightarrow Y$ from a metric space $X$ to a first-countable $T_{1}$-space $Y$ is spacious if and only if the collection $\mathscr{U}$ of all subsets of $X$ satisfies the condition of Theorem 1.6, and we therefore obtain the following corollary.

COROllary 1.7. If $f: X \rightarrow Y$ is a spacious, continuous map from a complete metric space $X$ onto a metrizable space $Y$, then $Y$ is completely metrizable.

A version of Corollary 1.7, for separable $X$ and $Y$ and with $f$ having compact fibers, was obtained by Ghoussoub and Maurey in [GM, Theorem I.1]. ${ }^{7}$ My effort to understand that result, and the realization that it did not appear to follow from known characterizations of complete metrizability, provided the original impetus for this paper.

After characterizing exhaustive covers in $\$ 2$, we prove Theorem 1.3 in $\$ 3$ and then establish the equivalence of Theorems 1.3 and 1.5 in $\S 4$. $\S 5$ and 6 prove Theorem 1.6 and Corollary 1.7. $\$ 7$ contains further results related to Theorem 1.5 , including two game-theoretic characterizations of spaces with a complete exhaustive sieve. $\S 8$, finally, compares the conditions in Theorems $1.2-1.5$ when $X$ is not necessarily metrizable.

\footnotetext{
${ }^{6}$ A set $A \subset X$ is $\varepsilon$-discrete if $d\left(x, x^{\prime}\right) \geqslant \varepsilon$ whenever $x, x^{\prime} \in A$ with $x \neq x^{\prime}$.

${ }^{7}$ While the statement of Theorem I.1 of [GM] does not explicitly assume that $f$ has compact fibers, the proof seems to require an interpretation of the definition which is tantamount to that assumption. I am indebted to I. Namioka for originally calling this to my attention.
} 


\section{A characterization of exhaustive covers.}

LEMMA 2.1. The following are equivalent for an indexed cover $\left(U_{\alpha}\right)_{\alpha \in A}$ of a space $X$.

(a) $\left(U_{\alpha}\right)_{\alpha \in A}$ is an exhaustive cover of $X$.

(b) The index set $A$ can be well-ordered such that $\cup_{\alpha^{\prime} \leqslant \alpha} U_{\alpha^{\prime}}$ is open in $X$ for all $\alpha \in A$.

Proof. (a) $\rightarrow$ (b). It suffices to show that, if $B \subset A$ with $\bigcup_{\beta \in B} U_{B}$ open in $X$, and if $B \neq A$, then there is an $\alpha \in A \backslash B$ such that $\left(\cup_{\beta \in B} U_{\beta}\right) \cup U_{\alpha}$ is also open in $X$. Let $S=X \backslash \cup_{\beta \in B} U_{\beta}$. If $S=\varnothing$, we can take $\alpha$ to be any element of $A \backslash B$. If $S \neq \varnothing$, pick any $\alpha \in A$ such that $U_{\alpha} \cap S$ is nonempty and relatively open in $S$; this $\alpha$ satisfies our requirements.

(b) $\rightarrow$ (a). Suppose $S \subset X$ is nonempty. Let $\alpha$ be the first element of $A$ such that $U_{\alpha} \cap S \neq \varnothing$. Then $U_{\alpha} \cap S=\left(\cup_{\alpha^{\prime} \leqslant \alpha} U_{\alpha^{\prime}}\right) \cap S$, so $U_{\alpha} \cap S$ is relatively open in $S$.

3. Proof of Theorem 1.3. If $X$ is completely metrizable, then $X$ has a complete sequence of open (hence exhaustive) cover by Theorem 1.2. Suppose, conversely, that $X$ has a complete sequence of exhaustive covers $\left(\mathscr{U}_{n}\right)$, and let us show that $X$ must be completely metrizable. Let $Y$ be any complete metric space containing $X$. By Theorem 1.1, it will suffice to show that $X$ is a $G_{\delta}$ in $Y$.

By Lemma 2.1, each $\mathscr{U}_{n}$ can be indexed as $\left\{U_{\alpha}: \alpha \in A_{n}\right\}$, with well-ordered $A_{n}$, such that $V_{\alpha}=\cup_{\alpha^{\prime} \leqslant \alpha} U_{\alpha^{\prime}}$ is open in $X$ for all $\alpha \in A_{n}$. Let $W_{\alpha}$ be the largest open subset of $Y$ whose intersection with $X$ is $V_{\alpha}$. For each $n$ and $\alpha \in A_{n}$, let $D_{\alpha}=$ $U_{\alpha} \backslash \cup_{\alpha^{\prime}<\alpha} U_{\alpha^{\prime}}$ and let $E_{\alpha}=\bar{D}_{\alpha} \cap W_{\alpha}$ (where the closure is taken in $Y$ ); note that $D_{\alpha} \subset E_{\alpha}$. Let $Z_{n}=\bigcup_{\alpha \in A_{n}} E_{\alpha}$ for all $n$, and let $Z=\bigcap_{n} Z_{n}$. We will show that each $Z_{n}$-and thus also $Z$-is a $G_{\delta}$ in $Y$, and that $X=Z$.

To show that $Z_{n}$ is a $G_{\delta}$ in $Y$, observe that $\left(W_{\alpha}\right)_{\alpha \in A_{n}}$ is an increasing family of open subsets of $Y$, and that $E_{\alpha} \subset W_{\alpha} \backslash \cup_{\alpha^{\prime}<\alpha} W_{\alpha^{\prime}}$ for all $\alpha \in A_{n}$ (for if $\alpha^{\prime}<\alpha$ in $A_{n}$, then $D_{\alpha} \cap V_{\alpha^{\prime}}=\varnothing$, hence $D_{\alpha} \cap W_{\alpha^{\prime}}=\varnothing$, so $\bar{D}_{\alpha} \cap W_{\alpha^{\prime}}=\varnothing$, and thus $E_{\alpha} \cap$ $\left.W_{\alpha^{\prime}}=\varnothing\right)$. Since $E_{\alpha}$ is a $G_{\delta}$ in $Y$ for all $\alpha \in A_{n}$, it follows from a result of D. Montgomery [Mo, Lemma 2] $]^{8}$ that $Z_{n}$ is also a $G_{\delta}$ in $Y$.

Let us now prove that $X=Z$. Since $X=\bigcup_{\alpha \in A_{n}} D_{\alpha} \subset \cup_{\alpha \in A_{n}} E_{\alpha}=Z_{n}$ for all $n$, we have $X \subset Z$. Suppose, conversely, that $y \in Z$, and let us show that $y \in X$. For all $n$ we have $y \in Z_{n}=\bigcup_{\alpha \in A_{n}} E_{\alpha}$, so $y \in E_{\alpha n}$, for some $a_{n} \in A_{n}$. Let $\mathscr{F}=\{V \cap$ $U_{\alpha_{n}}: V$ a neighborhood of $y$ in $\left.Y, n \geqslant 0\right\}$. Then $\mathscr{F}$ is a filter base on $X$ (since $y \in E_{\alpha_{n}} \subset \bar{U}_{\alpha_{n}}$ for all $n$ ) and each $U_{\alpha_{n}}$ contains some $F \in \mathscr{F}$, so some $x \in X$ is in $\bigcap\{\bar{F}: \stackrel{n}{F} \in \mathscr{F}\}$ because $\left(\mathscr{U}_{n}\right)$ is a complete sequence of covers of $X$. But clearly $\bigcap\{\bar{F}: F \in \mathscr{F}\}=\{y\}$ (because $Y$ is Hausdorff), so $y=x$ and hence $y \in X$.

4. Proof of Theorem 1.5. Theorem 1.5 follows immediately from Theorem 1.3 and the equivalence (a) $\leftrightarrow$ (b) in the following result. (For the nontrivial half of Theorem 1.5, we need only Theorem 1.3 and the implication $(b) \rightarrow(a)$.)

\footnotetext{
${ }^{8}$ This is the only part of our proof which depends on the metrizability of $X$. I am grateful to Roger Hansell and John Jayne for calling Montgomery's lemma and related results to my attention
} 
Proposition 4.1. The following are equivalent for a space $X$.

(a) $X$ has a complete sequence of exhaustive covers.

(b) $X$ has a complete exhaustive sieve.

(c) $X$ has a complete sieve $\left(\left\{U_{\alpha}: \alpha \in A_{n}\right\}, \pi_{n}\right)$, with well-ordered $A_{n}$ 's and order-preserving $\pi_{n}$ 's, such that $\cup_{\alpha^{\prime} \leqslant \alpha} U_{\alpha^{\prime}}$ is open in $X$ for all $\alpha \in A_{n}$ and all $n$.

(d) Same as (c), but requiring also that $\left\{U_{\alpha}: \alpha \in A_{n}\right\}$ is disjoint for all $n$.

Proof. (a) $\rightarrow$ (b). The analogous implication for open covers and sieves was proved in [M, Theorem 3.2, (a) $\rightarrow$ (b)], and essentially the same proof works in the present case.

(b) $\rightarrow$ (c). Let $\left(\left\{U_{\alpha}: \alpha \in A_{n}\right\}, \pi_{n}\right)$ be a complete exhaustive sieve on $X$. We will show that the $A_{n}$ 's can be well-ordered to satisfy (c). Since $U_{\alpha}=X$ for all $\alpha \in A_{0}$, we can well-order $A_{0}$ arbitrarily. Suppose $A_{0}, \ldots, A_{n}$ have been suitably ordered. For each $\alpha \in A_{n}$, apply Lemma 2.1 to well-order $\pi_{n}^{-1}(\alpha)$ so that $\bigcup\left\{U_{\beta^{\prime}}: \beta^{\prime} \in \pi_{n}^{-1}(\alpha)\right.$, $\left.\beta^{\prime} \leqslant \beta\right\}$ is open in $U_{\alpha}$ for all $\beta \in \pi_{n}^{-1}(\alpha)$. Well-order $A_{n+1}$ by letting $\beta^{\prime}<\beta$ if either $\pi_{n}\left(\beta^{\prime}\right)<\pi_{n}(\beta)$ in $A_{n}$ or if $\pi_{n}\left(\beta^{\prime}\right)=\pi_{n}(\beta)=\alpha$ and $\beta^{\prime}<\beta$ in $\pi_{n}^{-1}(\alpha)$. It is easy to check that this works.

(c) $\rightarrow$ (d). Let $\left(\left\{U_{\alpha}: \alpha \in A_{n}\right\}, \pi_{n}\right)$ be as in (c). For each $n$ and $\alpha \in A_{n}$, let $U_{\alpha}^{*}=U_{\alpha} \backslash\left(U_{\alpha^{\prime}<\alpha} U_{\alpha^{\prime}}\right)$. It is easy to check that $\left(\left\{U_{\alpha}^{*}: \alpha \in A_{n}\right\}, \pi_{n}\right)$ is a sieve on $X$ satisfying (d). (To show that $U_{\beta}^{*} \subset U_{\alpha}^{*}$ whenever $\beta \in \pi_{n}^{-1}(\alpha)$, one needs the assumption that $\pi_{n}$ is order-preserving.)

(d) $\rightarrow$ (a). Let $\left(\left\{U_{\alpha}: \alpha \in A_{n}\right\}, \pi_{n}\right)$ be a sieve on $X$ satisfying (d), and let $\mathscr{U}_{n}=\left\{U_{\alpha}\right.$ : $\left.\alpha \in A_{n}\right\}$. Then each $\mathscr{U}_{n}$ is an exhaustive cover of $X$ by Lemma 2.1, (b) $\rightarrow$ (a). To show that $\left(\mathscr{U}_{n}\right)$ is a complete sequence of covers, let $\mathscr{F}$ be a filter base on $X$ such that each $\mathscr{U}_{n}$ has an element $U_{\alpha_{n}}\left(\alpha_{n} \in A_{n}\right)$ containing some $F \in \mathscr{F}$. Then $U_{\alpha_{n+1}} \cap$ $U_{\alpha_{n}} \neq \varnothing$ for all $n$, and hence (since $\mathscr{U}_{n}$ is disjoint) $\pi_{n}\left(\alpha_{n+1}\right)=\alpha_{n}$. Since $\left(\left\{U_{\alpha}\right.\right.$ : $\left.\left.\alpha \in A_{n}\right\}, \pi_{n}\right)$ is complete, it follows that $\bigcap\{\bar{F}: F \in \mathscr{F}\} \neq \varnothing$.

It should be remarked that the analogue of the implication (b) $\rightarrow$ (a) for open sieves and covers is generally false [C $\check{C} \mathbf{N}$, Example 2.4], although it is true if $X$ is paracompact [M, Theorem 3.2, (b) $\rightarrow$ (a)].

5. Proof of Theorem 1.6. By Theorem 1.5, it will suffice to show that $Y$ has a complete exhaustive sieve. By induction, choose indexed subcollections $\left\{U_{\alpha}: \alpha \in A_{n}\right\}$ of $\mathscr{U}$ and maps $\pi_{n}: A_{n+1} \rightarrow A_{n}$ such that $\left\{U_{\alpha}: \alpha \in A_{0}\right\}=\{X\}$ and, for all $n$ and all $\alpha \in A_{n},\left\{U_{\beta}: \beta \in \pi_{n}^{-1}(\alpha)\right\}$ is the collection of all $1 /(n+1)$-modest ${ }^{9}$ subsets of $U_{\alpha}$ which are elements of $\mathscr{U}$. Let $V_{\alpha}=f\left(U_{\alpha}\right)$. The hypothesis of our theorem implies that $\left(\left\{V_{\alpha}: \alpha \in A_{n}\right\}, \pi_{n}\right)$ is an exhaustive sieve on $Y$, so we need only show that this sieve is complete.

Suppose that $\alpha_{n} \in A_{n}$ with $\pi_{n}\left(\alpha_{n+1}\right)=\alpha_{n}$ for all $n$, and that $\mathscr{F}$ is a filter base on $Y$ such that each $V_{\alpha_{n}}$ contains some $F \in \mathscr{F}$, and let us check that $\bigcap\{\bar{F}: F \in \mathscr{F}\} \neq \varnothing$. Let $\mathscr{E}=\left\{f^{-1}(F) \cap U_{\alpha_{n}}: F \in \mathscr{F}, n \geqslant 0\right\}$. Then $\mathscr{E}$ is a filter base on $X$ and each $U_{\alpha_{n}}$ contains an $E \in \mathscr{E}$, so there is an $x \in \bigcap\{\bar{E}: E \in \mathscr{E}\}$ by [K, Corollary, p. 412]. But then $f(x) \in \bigcap\{\bar{F}: F \in \mathscr{F}\}$, which completes the proof.

\footnotetext{
${ }^{9} \varepsilon$-modest spaces are defined in the introduction, just before Theorem 1.6.
} 
6. Proof of Corollary 1.7. We begin with a characterization of spacious maps. ${ }^{10}$

LEMMA 6.1. The following are equivalent for a map $f: X \rightarrow Y$ from a metric space $(X, d)$ to a first-countable $T_{1}$-space $Y$.

(a) $f$ is spacious.

(b) For all $\varepsilon>0$, every nonempty $E \subset X$ has a nonempty, $\varepsilon$-modest subset $S$ such that $f(S)$ is open in $f(E)$.

(c) For all $\varepsilon>0$ and $E \subset X,\{f(S): S \subset E, S$ is $\varepsilon$-modest $\}$ is an exhaustive cover of $f(E)$.

Proof. (a) $\rightarrow$ (b). Suppose (b) is false for some $\varepsilon>0$ and $E \subset X$. Then there is no $\varepsilon$-modest $S \subset E$ such that $f(S)$ contains a nonempty open subset of $f(E)$. We will show that (a) must be false by picking a $\frac{1}{2} \varepsilon$-discrete sequence $x_{n} \in E$ such that the set $\left\{f\left(x_{n}\right): n \in \omega\right\}$ has no isolated point.

For each $y \in f(E)$, let $\left(V_{n}(y)\right)$ be a countable base at $y$ in $f(E)$. Pick $u: \omega \rightarrow \omega$ such that $u(n)<n$ for all $n>0$ and $u^{-1}(n)$ is infinite for all $n \in \omega$. It will suffice to choose $x_{n} \in E$ inductively such that, letting $S_{n}=\cup_{m<n}\left\{x \in E: d\left(x, x_{m}\right)<\frac{1}{2} \varepsilon\right\}$ and $W_{n}=V_{n}\left(f\left(x_{u(n)}\right)\right)$, we have $f\left(x_{n}\right) \in W_{n} \backslash f\left(S_{n}\right)$ for all $n>0$. But that is easily done, because $S_{n}$ is $\varepsilon$-modest and hence $f\left(S_{n}\right) \not \supset W_{n}$ by our assumption.

(b) $\rightarrow$ (a). Let $\varepsilon>0$, and let $E \subset X$ be nonempty and $\varepsilon$-discrete. By (b) there is a nonempty, $\frac{1}{2} \varepsilon$-modest $S \subset E$ with $f(S)$ open in $f(E)$. Since $E$ is $\varepsilon$-discrete, $S$ must be finite, so $f(S)$ is finite and hence every $y \in f(S)$ is an isolated point of $f(E)$.

(b) $\rightarrow$ (c). Suppose $T \subset f(E)$ is nonempty. Let $E^{\prime}=E \cap f^{-1}(T)$. Then $E^{\prime} \neq \varnothing$, so there is a nonempty, $\varepsilon$-modest $S \subset E^{\prime} \subset E$ such that $f(S)$ is open in $T$.

(c) $\rightarrow$ (b). Clear.

Proof of Corollary 1.7. Let $f: X \rightarrow Y$ be as in Corollary 1.7. By Lemma 6.1, (a) $\rightarrow$ (c), $f$ satisfies the hypothesis of Theorem 1.6 with $\mathscr{U}$ the collection of all subsets of $X$. Hence $Y$ is completely metrizable by Theorem 1.6.

7. Some analogues of Theorem 1.5. The results in this section are somewhat peripheral to the main purpose of this paper, and will therefore mostly be stated without proof.

Our first result gives a characterization of complete metrizability which is even weaker than that in Theorem 1.5. For any collection $\mathscr{U}$ of subsets of $X$, let $\mathscr{U}^{*}$ denote the collection of all subsets of finite unions of elements of $\mathscr{U}$.

THEOREM 7.1. The following are equivalent for a metrizable space $X$.

(a) $X$ is completely metrizable.

(b) $X$ has a complete sieve $\left(\left\{U_{\alpha}: \alpha \in A_{n}\right\}, \pi_{n}\right)$ such that, for all $n$ and $\alpha \in A_{n}$, $\left\{U_{\beta}: \beta \in \pi_{n}^{-1}(\alpha)\right\}^{*}$ is an exhaustive cover of $U_{\alpha}$.

\footnotetext{
${ }^{10}$ These maps are defined in the introduction before Corollary 1.7.
} 
Let us call $\mathscr{U}$ a strictly exhaustive cover of $X$ if every nonempty $S \subset X$ has a nonempty, relatively open subset $U \in \mathscr{U}$; a strictly exhaustive sieve is now defined by the obvious modification of the definition of an exhaustive sieve. Evidently every strictly exhaustive cover or sieve is exhaustive, and it is easy to show that every space with a complete exhaustive sieve also has a complete, strictly exhaustive sieve. Observe that open covers and sieves, while always exhaustive, are generally not strictly exhaustive. Nevertheless, strictly exhaustive sieves have a virtue not possessed by exhaustive sieves: When used to characterize completely metrizable spaces, they allow the completeness requirement on the sieve to be weakened and simplified. Let us call a sieve $\left(\left\{U_{\alpha}: \alpha \in A_{n}\right\}, \pi_{n}\right)$ on $X$ pseudo-complete if, whenever $\alpha_{n} \in A_{n}$ with $\pi_{n}\left(\alpha_{n+1}\right)=\alpha_{n}$ for all $n$, then $\bigcap_{n} \bar{U}_{\alpha_{n}} \neq \varnothing$. Clearly every complete sieve is pseudo-complete.

THEOREM 7.2. The following are equivalent for a metrizable space $X$.

(a) $X$ is completely metrizable.

(b) X has a pseudo-complete, strictly exhaustive sieve.

It should be remarked that "strictly exhaustive" cannot be weakened to "exhaustive" in Theorem 7.2, since every space $X$ has a pseudo-complete exhaustive sieve; it suffices to take $U_{\alpha}=X$ for all $\alpha$.

We now turn to the final topic of this section. F. Topsøe [T, Theorem 9] has given a game-theoretic characterization of spaces which have a complete open sieve. Let us indicate how this can also be done for spaces having a complete exhaustive sieve.

Let $G(X)$ be the following two-person game. Players I and II alternately choose nonempty subsets $S_{1} \supset T_{1} \supset S_{2} \supset T_{2} \supset \cdots$ of $X$ such that $T_{n}$ (chosen by II) is relatively open in $S_{n}$ (chosen by I) for all $n$. Player II wins if, whenever $\mathscr{F}$ is a filter base on $X$ such that each $T_{n}$ contains some $F \in \mathscr{F}$, then $\cap\{\bar{F}: F \in \mathscr{F}\} \neq \varnothing$.

In the following result, a strategy for Player II assumes that he has a memory, so that his $n$th move $T_{n}$ may depend on the first $n$ moves $S_{1}, \ldots, S_{n}$ of Player I. A stationary strategy, on the other hand, assumes no memory, and is therefore simply a function $\phi$ which assigns to every nonempty $S \subset X$ a nonempty, relatively open $\phi(S) \subset S$; using this $\phi$, Player II chooses $T_{n}=\phi\left(S_{n}\right)$.

THEOREM 7.3. The following are equivalent for any space $X$.

(a) X has a complete, exhaustive sieve.

(b) Player II has a stationary winning strategy for $G(X)$.

(c) Player II has a winning strategy for $G(X)$.

Proof. (a) $\rightarrow$ (b). By Lemma 4.1, $X$ has a complete sequence $\left(\mathscr{U}_{n}\right)$ of exhaustive covers, and we may suppose that $\mathscr{U}_{n+1}$ refines $\mathscr{U}_{n}$ for all $n$.

Suppose $\varnothing \neq S \subset X$. Let $k(S)$ be the first $n$ such that $S$ is not a subset of any $U \in \mathscr{U}_{n}$; if there is no such $n$, let $k(S)=\infty$. If $k(S)<\infty$, pick $U(S) \in \mathscr{U}_{k(S)}$ such that $U(S) \cap S$ is nonempty and relatively open in $S$, and define $\phi(S)=U(S) \cap S$; if $k(S)=\infty$, define $\phi(S)=S$ 
Let us show that the strategy $T_{n}=\phi\left(S_{n}\right)$ wins for Player II. Observe first that, if $k\left(S_{n}\right)<\infty$, then $k\left(S_{n+1}\right)>k\left(S_{n}\right)$; in fact, $k\left(S_{n+1}\right) \geqslant k\left(S_{n}\right)$ because $S_{n+1} \subset S_{n}$, and $k\left(S_{n+1}\right) \neq k\left(S_{n}\right)$ because $S_{n+1} \subset \phi\left(S_{n}\right) \subset U\left(S_{n}\right) \in \mathscr{U}_{k\left(S_{n}\right)}$. Hence $k\left(S_{n}\right) \geqslant n$ for all $n$, and it follows that $T_{n} \subset U_{n}$ for some $U_{n} \in \mathscr{U}_{n}$. Now if $\mathscr{F}$ is a filter base on $X$ such that each $T_{n}$ contains some $F_{n} \in \mathscr{F}$, then also $U_{n} \supset F_{n}$, and therefore $\cap\{\bar{F}: F \in \mathscr{F}\} \neq \varnothing$.

(b) $\rightarrow$ (c). Clear.

(c) $\rightarrow$ (a). We follow the first half of the proof of [T, Theorem 9]. Let $A_{0}=\left\{\alpha_{0}\right\}$ and $U_{\alpha_{0}}=X$. For $n>0$, let $A_{n}$ be the set of all $\left(S_{1}, T_{1}, \ldots, S_{n}, T_{n}\right)$ of first $n$ moves by Players I and II which can occur in a game in which Player II employs his winning strategy, and define $\pi_{n}: A_{n+1} \rightarrow A_{n}$ by $\pi_{n}\left(S_{1}, T_{1}, \ldots, S_{n+1}, T_{n+1}\right)=$ $\left(S_{1}, T_{1}, \ldots, S_{n}, T_{n}\right)$. For $\alpha=\left(S_{1}, T_{1}, \ldots, S_{n}, T_{n}\right) \in A_{n}$, let $U_{\alpha}=T_{n}$. This defines a complete (strictly) exhaustive sieve on $X$.

Consider, next, the following game $G^{*}(X)$, which is even simpler than $G(X)$. The moves for $G^{*}(X)$ are the same as for $G(X)$, but Player II wins $G^{*}(X)$ if merely $\bigcap_{n} \bar{T}_{n} \neq \varnothing$.

THEOREM 7.4. A space $X$ has a pseudo-complete, strictly exhaustive sieve if and only if Player II has a winning strategy for $G^{*}(X)$.

I do not know whether the conditions in Theorem 7.4 always imply that Player II has a stationary winning strategy for $G^{*}(X)$.

COROLlaRY 7.5. The following are equivalent for a metrizable space $X$.

(a) $X$ is completely metrizable.

(b) Player II has a stationary winning strategy for $G(X)$.

(c) Player II has a winning strategy for $G(X)$.

(d) Player II has a stationary winning strategy for $G^{*}(X)$.

(e) Player II has a winning strategy for $G^{*}(X)$.

Proof. We have $(a) \leftrightarrow(b) \leftrightarrow$ (c) by Theorems 1.5 and 7.3 , and (a) $\leftrightarrow$ (e) by Theorems 7.2 and 7.4. Since clearly (b) $\rightarrow$ (d) $\rightarrow$ (e), our assertion follows.

Consider, finally, the modification $G_{0}(X)$ of $G(X)$ where all moves $S_{1} \supset T_{1} \supset S_{2}$ $\supset T_{2} \supset \cdots$ are required to be open in $X$. This game, which is more restrictive for Player I than $G(X)$, no longer serves to characterize complete metrizability. Indeed, any metrizable space $X$ with a dense, completely metrizable subspace gives Player II a (stationary) winning strategy for $G_{0}(X)$, but such a space need not be completely metrizable.

In terms of sieves, Player II has a winning strategy for $G_{0}(X)$ if and only if $X$ has a structure $\left(\left\{U_{\alpha}: \alpha \in A_{n}\right\}, \pi_{n}\right)$ with all the properties of a complete open sieve except that $\bigcup_{\alpha \in A_{n}} U_{\alpha}$ is only dense in $X$ for all $n$ and $\cup\left\{U_{\beta}: \beta \in \pi_{n}^{-1}(\alpha)\right\}$ is only dense in $U_{\alpha}$ for all $\alpha \in A_{n}$. As observed by I. Namioka, if $\left(\left\{U_{\alpha}: \alpha \in A_{n}\right\}, n\right)$ is a complete exhaustive sieve on $X$, then $\left(\left\{U_{\alpha}^{\circ}: \alpha \in A_{n}\right\}, \pi_{n}\right)$ is such a structure on $X$. 
8. The conditions of Theorems 1.2-1.5. The conditions in Theorems $1.2-1.5$ are all equivalent in metrizable spaces. Without metrizability, their relationships are summarized by the following diagram, where each condition is indicated by the number of the theorem in which it appears.

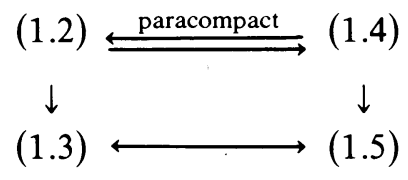

The relations between (1.2) and (1.4) were established in [M, Theorem 3.2], the equivalence between (1.3) and (1.5) was proved in Proposition 4.1, and the vertical implications are obvious. Corollary 8.3 below indicates some obstacles to finding conditions, other than metrizability, under which the vertical implications can be reversed. We recall that a completely regular space satisfies (1.2) if and only if it is Čech-complete $^{11}[\mathbf{A r}, \mathbf{F}]$, and that a space satisfying (1.4) is called sieve-complete in $[\mathbf{M}] .^{12}$

LEMMA 8.1. Every scattered space $X$ satisfies the condition of Theorem 1.3.

Proof. This was already observed after the statement of Theorem 1.3.

Lemma 8.2. If $X$ is Čech-complete, and if $\{x\}$ is a $G_{\delta}$ in $X$, then $X$ has a countable base at $x$.

Proof. Let $X^{*}$ be a Hausdorff compactification of $X$. Then $X$ is a $G_{\delta}$ in $X^{*}$, so $\{x\}$ is a $G_{\delta}$ in $X^{*}$, hence $X^{*}$ (being compact) has a countable base at $x$, and therefore $X$ also has a countable base at $x$.

COROllary 8.3. Suppose that $X$ is a countable $T_{1}$-space with a unique nonisolated point $x_{0}$, and that $X$ does not have a countable base at $x_{0}$. Then $X$ is a paracompact space which satisfies the condition of Theorem 1.3, but $X$ is not Cech-complete and not sieve-complete.

Proof. By Lemma 8.1, $X$ satisfies the condition of Theorem 1.3. By Lemma 8.2, $X$ is not Čech-complete. Since $X$ is countable and regular, it is paracompact. Since paracompact, sieve-complete spaces are Čech-complete, $X$ is not even sieve-complete.

\footnotetext{
${ }^{11} \mathrm{~A}$ completely regular space is $\check{C}$ ech-complete if it is a $G_{\delta}$ in one (equivalently, in all) of its Hausdorff compactifications.

12 These spaces and closely related ones had previously been studied, under different names, in [C $\check{C} \mathbf{N}$, $\mathbf{W}$ and $\mathbf{W W}$ ].
} 


\section{REFERENCES}

[A] P. Alexandrov, Sur les ensembles de la première classe et les espaces abstraits, C. R. Acad. Sci. Paris Sér. I. Math. 178 (1924), 185-187.

[Ar] A. V. Arhangel'skī̌, On topological spaces complete in the sense of Čech, Vestnik Moskov. Univ. Ser. I. Mat. Mekh. 2 (1961), 37-40. (Russian)

[CČN] J. Chaber, M. M. Čoban and K. Nagami, On monotone generalizations of Moore spaces, Čech complete spaces and p-spaces, Fund. Math. 84 (1974), 107-119.

[F] Z. Frolik, Generalizations of the $G_{\delta}$-property of complete metric spaces, Czechoslovak Math. J. 10 (1960), 359-379.

[GM] N. Ghoussoub and B. Maurey, $G_{\delta}$-embeddings in Hilbert space, J. Funct. Anal. 61 (1985), $72-97$.

$\left[\mathbf{H}_{1}\right]$ F. Hausdorff, Die Mengen $G_{\delta}$ in vollständigen Räumen, Fund. Math. 6 (1924), 146-148.

$\left[\mathrm{H}_{2}\right]$, Über innere Abbildungen, Fund. Math. 23 (1934), 279-291.

[K] K. Kuratowski, Topologv, vol. 1, Academic Press, New York, 1966.

[M] E. Michael, Complete spaces and tri-quotient maps, Illinois J. Math. 21 (1977), 716-733.

[Mo] D. Montgomery, Non-separable metric spaces, Fund. Math. 25 (1935), 527-533.

[T] F. Topsøe, Topological games and Čech completeness, Proc. Fifth Prague Topological Symposium, 1981, Sigma Series in Pure Math., vol. 3, Heldermann, Berlin, 1982, pp. 613-630.

[W] H. H. Wicke, Open continuous images of certain kinds of $M$-spaces and completeness of mappings and spaces, General Topology Appl. 1 (1971), 85-100.

[We] N. Wedenissoff, Sur les espaces metriques complets, J. Math. Pures Appl. (9) 9 (1930), 377-381.

[WW] H. H. Wicke and J. M. Worrell, Jr., On the open continuous images of paracompact Čech complete spaces, Pacific J. Math. 37 (1971), 265-276.

Department of Mathematics, University of Washington, Seattle, Washington 98195 